\title{
Structural neuroimaging and neuropsychological correlates of a single case of focal central pontine myelinolysis: Intact memory function with decreased intellectual and motor functions
}

\author{
Myriah K. Mulvogue ${ }^{1}$, Thomas P. Robinson', Mohammad S. Ijaz ${ }^{3}$, Simon M. McCrea ${ }^{4}$ \\ ${ }^{1}$ Department of Psychology, University of Regina, Regina, Canada \\ ${ }^{2}$ Functional Rehabilitation Program, Wascana Rehabilitation Centre, Regina, Canada \\ ${ }^{3}$ Division of Neurology, Regina General Hospital, Regina, Canada \\ ${ }^{4}$ Director-Neuropsychology Service, Wascana Rehabilitation Centre, Regina, Canada \\ Email: ${ }^{*}$ simon.mccrea@rqhealth.ca
}

Received 5 August 2012; revised 15 September 2012; accepted 2 October 2012

\begin{abstract}
We report on the case of middle-aged right-handed woman with central pontine myelinolysis (CPM) revealed by high resolution structural $\mathrm{T} 2$-weighted FLAIR MRI imaging. There was a general flattening of Wechsler Adult Intelligence Scale-Fourth Edition subtest scores which were 1 standard deviation below expected values. In contrast Wechsler Memory Scale -Fourth Edition visual and auditory memory scores remained within the normal range. Verbal working memory appeared mildly impaired while nonverbal working memory was not. Scores on the Advanced Clinical Solution's Social Perception battery were all in the normal range as were academic skills measured by the Wide Range Achievement Test-Fourth Edition. Performance was impaired on the Delis-Kaplan Executive Function System's counterpart of the TrailMaking Test: Part B. Similarly, on the Draw-A-Person Test there was a discrepancy in that our patient's standard score was 76 compared to her estimated premorbid FSIQ in the average range. She also displayed bilateral motor coordination slowing on the Finger Tapping task collectively suggesting damage to pontine motor tracts. The Minnesota Multiphasic Personality Inventory-Second Edition-Restructured Form profile was consistent with a diagnosis of severe anxiety and depression perhaps due to damage to serotoninergic neural tracts originating within the central pons. Finally, the patient displayed severe sleep disturbances and other signs of reticular activating formation injury. CPM may constitute a unique means of studying reversible subcortical lesions in the central pons in otherwise healthy subjects with benign
\end{abstract}

"Corresponding author. illness. To our knowledge this is among the first patients with CPM without the usual risk factors for the disorder and who was otherwise healthy. Knowledge of the etiology and neuropsychology of such patients might aid in understanding the interaction of the fronto-ponto-cerebellar tracts in executive functions and motor programming.

Keywords: Central Pontine Myelinolysis; Neuropsychology; T2-Weighted Structural MRI FLAIR Imaging; Motor Functions; Subcortical Signs; Fronto-Ponto-Cerebellar Function

\section{INTRODUCTION}

Central Pontine Myelinolysis (CPM) is a rare neurologic disorder associated with demyelination of the central pons. It was originally named to describe the appearance of a "batwing" shaped lesion of the central pons in patients with a history of alcohol abuse or malnourishment [1]. Indeed, chronic alcoholism continues to be among the most commonly associated disorders associated with CPM [2]. Since the initial discovery in patients with histories of alcoholism, similar neuropathology has been recorded in patients with electrolyte disturbances. With the advent of high resolution structural MRI more relatively benign cases of CPM are being increasingly discovered. In the past before computed tomography, these cases were only definitively identified at autopsy and CT scans are not particularly good at visualizing CPM in the post-acute phase compared with MRI [3]. In particular, in patients with hypo- and, more rarely, hypernatremia, demyelinization may occur with attempts to quickly normalize cerebral fluid sodium levels. This appears to lead to significant cerebral edema and dehydration of 
brain cells within the central pons [4-7]. Lampl and Yazdi (2002) note in their review that extracellular osmotic dysregulation results in the myelin sheath being stripped from the axon [2] such that oligodendrocytes are damaged [8] resulting in myelin toxic substances being released from vascular endothelial cells [9].

Although the exact etiology remains unknown, a significant number of CPM cases are also associated with liver transplantation, [2] particularly in those patients experiencing complications such as sepsis, hypoxia, and hepatic encephalopathy immediately post-transplant [10]. Some evidence suggests that cyclosporine, an immunosuppressive agent, may be causally related in transplant patients with CPM $[11,12]$. Liver transplant patients also account for the vast majority of childhood cases of CPM, for which no causal explanation has been supported thus far [13]. Hence, dysregulation of sodium ion metabolism and concomitant neurotoxicity of brain cells within the central pons appears to play a key role in most hypothesizing about the etiology of this disorder. However, cases with an absence of these comorbidities, but with the presence of other disorders associated with serum sodium imbalances including malignant tumours, pulmonary infections, diabetes mellitus and epilepsy have also been documented $[2,14,15]$.

Case reports outlining the clinical manifestation of CPM suggest diverse clinical neurological symptoms across individual patients. Motor symptoms such as spastic quadriparesis, dysarthria, and pseudobulbar palsy are widely reported [16-18]. Global cognitive dysfunctions, including those associated with executive functions, attention and memory deficits, increased disinhibition, and general disorientation have also been regularly documented. Detailed neuropsychological studies of the cognitive correlates of CPM however have been quite sparse and what studies that have been completed often have assessed only a few isolated domains of cognitive functioning [16,19-21]. There are few or no published cases of CPM studied with comprehensive neuropsychological assessments with a few exceptions. One intensively studied CPM case found that all neuropsychological deficits were fully reversible four months after the acute stage [16]. Finally, CPM patient's may display emotional and mood symptoms such as pathological crying, or emotional lability [21] as well as personality changes [22].

\section{METHOD}

In this study we comprehensively describe a patient with central pontine myelinolysis with cognitive ability, memory, motor, emotion, mood and social cognition measures for research and archival purposes. High resolution structural MRI was also examined. The features of the disorder were classically CPM with the archetypal batwing lesion localized within the central pons. There were little additional extrapontine findings thereby providing an ideal means of studying the influence of a small welllocalized central pontine lesion. Although memory functions were unimpaired intellectual and motor functions were mildly impaired and we explain these findings on the basis of the highly focalized nature of the lesion within the vicinity of the medial raphe nucleus. The patient completed a comprehensive battery of clinical neuropsychological tests that is detailed elsewhere [23]. This neuropsychological evaluation was directed at examining a variety of functions of the central nervous system with a focus on higher cortical functions. Formal assessment of mood, affect, personality, and interpersonal factors was also carried out. Response validity was assessed using Advanced Clinical Solutions Effort Assessment which indicated the parametres were acceptable. The patient was administered all 15 subtests of the WAIS-IV in order to obtain an accurate level of general intellectual functioning as well as to examine ipsative differences in subtest score profiles. See Table 1.

\section{RESULTS}

A middle-aged right-handed woman, was initially referred to the Neuropsychology Service for problems with memory, persistent headaches, and symptoms of anxiety and depression that had developed over several years. The referral was made in order to determine whether the patient's mood symptoms were affecting her cognitive functions or whether her apparent cognitive decline was

Table 1. Wechsler adult intelligence scale - fourth edition (WAIS-IV) scores. A standard score has a mean of 100 and a standard deviation of 15 .

\begin{tabular}{ccccc}
\hline WAIS-IV Scales & IQ Score & 95\% Confidence Interval & Percentile & Qualitative Description \\
\hline Verbal Comprehension & 92 & $86-99$ & 30 th & Average \\
Perceptual Reasoning & 90 & $84-97$ & 25 th & Average \\
Verbal Working Memory & 82 & $76-91$ & 12 th & Low Average \\
Processing Speed & 91 & $84-100$ & 27 th & Average \\
Full Scale IQ & 85 & $81-90$ & 16 th & Low Average \\
General Ability Index & 89 & $84-94$ & 23 rd & Low Average \\
\hline
\end{tabular}


due to neurological factors. Upon assessment, the patient reported language-related difficulties including problems with expressing her thoughts, finding the right words, and understanding what others were saying or what she had read. In addition, she reported difficulties with balance and excessive fatigue, difficulties being able to think as quickly as needed, losing her train of thought, and general confusion and disorientation. She also reported significant difficulties with memory including forgetting facts, appointments and the order of events. However, she reported no difficulties with procedural memory. She also described experiencing ringing in her ears. Along with the insidious onset of these intellectual difficulties the patient noted she was experiencing changes in her mood, behaviour, and personality. Specifically, she stated she had been experiencing periods of severe sadness, anxiety, and euphoria, and increased emotional reactivity, lability, and apathy. Further, the patient expressed concern about disinhibition, lowered frustration tolerance, an inability to stay asleep at night, the presence of visual hallucinations and an increase in automatic-types of behaviors.

Medical review included a past history of depression and anxiety as well as stress, fatigue, and migraines. A structural MRI revealed an amorphous central non-enhancing increased T2-weighted FLAIR signal area in the central pons, along with tiny bifrontal non-enhancing areas of periventricular white matter increased signal in the centrum semiovale. The spatial configuration and location of the lesion was highly consistent with previously described central pontine myelinolysis - the lesion was adjacent to the fourth ventricle within the pons and revealed the characteristic pontine batwing shape [1]. The patient reported being once admitted to hospital overnight for severe headaches. She rarely or never drank alcohol. The patient was concurrently prescribed venlafaxine, topiramate, bromocriptine, rabaprazole, and amitrypyline. Of note, the patient's family history included two fatal heart-related problem and strokes. Sodium levels were within normal limits near the time of the neuropsychological evaluation $(140 \mathrm{mmol} / \mathrm{L})$.

Upon initial observation, the patient appeared drowsy despite reporting sleeping for nine hours the previous evening. She complained of waking often and being unable to fall back asleep sometimes as many as four times per evening. The patient made appropriate eye contact, but displayed dullness in her facial muscle expressivity. Motor activity appeared normal, although somewhat slowed. She displayed a mild right-sided paresis of the lips and mouth. The patient reported experiencing symptoms consistent with orthostatic hypotension, including light-headedness and balance difficulties. The patient described her mood over the past two weeks as flat and absent. Upon observation the patient sounded depressed
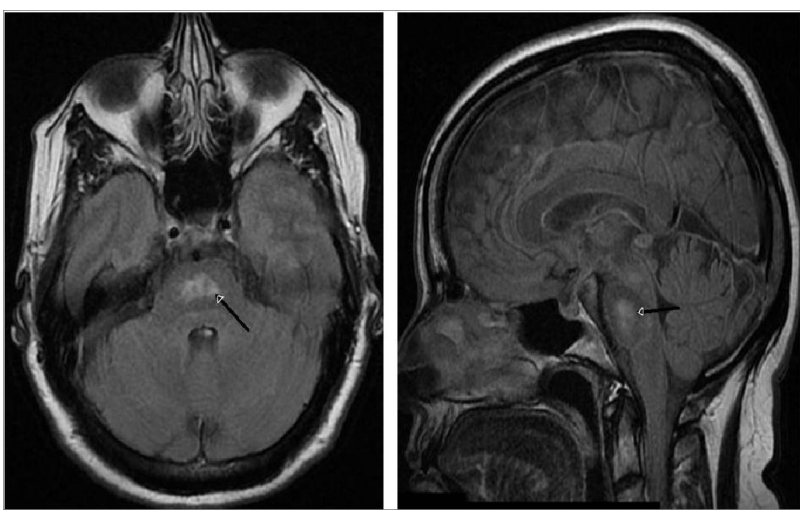

Figure 1. T2-weighted axial FLAIR image through the medial pons (left). T2-weighted sagittal FLAIR image through the pons (right). Black arrows denotes the center of the mass of the hyperintensity.

with flattened affect, with an absence of clearly expressed emotion. Intensity of affect was blunted and range of affect was restricted, particularly in response to positive cues. The patient demonstrated verbose and inaccurate speech with significant word-finding difficulties. Repetition of complex sentences was normal, as was comprehension of three-step commands and the patient was able to successfully define abstract concepts. She stated that while reading she would involuntarily skip words and lose focus. Her prosody was monotone. The patient scored within an average to low-average range on all composite scale and subtest scores, with a Full Scale IQ score of 85 (16th percentile).

Premorbid intellectual functioning was assessed using the Advanced Clinical Solutions Test of Premorbid Functioning. The patient's estimated premorbid Full Scale IQ was $106(95 \%$ confidence interval $=82-130)$. As the patient had completed a four year baccalaureate degree and successfully worked in middle management for many years, the predicted score of 106 was likely an accurate low-bound estimate of premorbid ability. Hence, there was a minimum 1 standard deviation unit level of mild intellectual impairment. At the WAIS-IV subtest level Figure Weights was in the impaired range whereas Picture Completion was unimpaired. The difference between Figure Weights and Picture Completion was significantly different $(\Delta=5$, critical value $=2.99, \mathrm{p}<0.05)$. Picture Completion is traditionally considered to be one of the best "hold" measures in the WAIS-R and WAIS-III batteries and is among the subtests least affected by brain damage [24]. Conversely, the Figure Weights subtest has the highest loading on the general factor in the WAIS-IV [25] indirectly supporting the hypothesis of the involvement of many brain systems for performance on this task. A corollary of this truism would be that it would be among the subtests most affected by brain damage [23]. See 
Table 2.

The Brief Cognitive Status Examination (BCSE) of the WMS-IV was administered to test the patient's orientation to time, mental control, constructional apraxia, incidental memory recall, inhibitory control and verbal generativity. The patient scored in the Very Low (80) and impaired level of functioning on the BCSE. Time estimation appeared to be particularly impaired. The Brief Cognitive Status Examination is analogous to the MiniMental Status Examination [26] and is purported to be a useful measure to evaluate global cognitive functioning. Conversely, the patient scored in the high average to average range on all WMS-IV Index scores (Table 3).

Performance on Visual Memory Index (117) was significantly greater than expected based on an obtained General Ability Index (89) $(\Delta=23$, critical value $=7.23$, $p<0.05$, base rate in standardization sample $=3 \%$ ). The Visual Working Memory Index of 103 was marginally significantly greater than the Verbal Working Memory Index of $82(\Delta=21, \mathrm{z}=1.40, \mathrm{p}<0.10)$ perhaps suggestive of lateralized findings. The lateralized working memory finding might be congruent with mild right sided facial paresis. Scores on both the verbal and nonverbal memory subtests were in the Average to Superior range.

Conversely there were a number of significant motor findings. The Finger Tapping test is a measure of selfdirected manual motor speed. The patient's Finger Tapping scores were in the extremely low and impaired range. Grip Strength is a measure of the intensity of voluntary grip movements [27]. The patient's grip strength was in the very low but unimpaired ranges for both hands. See Table 4. Similarly, performance on the

Table 2. Wechsler adult intelligence scale - fourth edition (WAIS-IV) subtest scores. An age scaled score has a mean of 10 and a standard deviation of 3 . VCI $=$ Verbal Comprehension Index, PRI = Perceptual Reasoning Index, Verbal WMI = Verbal Working Memory Index, PSI = Processing Speed Index.

\begin{tabular}{|c|c|c|c|}
\hline WAIS-IV Subtests (VCI) & Age Scaled Score & Percentile & Qualitative Description \\
\hline Similarities & 7 & 16 & Low Average \\
\hline Vocabulary & 10 & 50 & Average \\
\hline Information & 9 & 37 & Average \\
\hline $\begin{array}{c}\text { Comprehension } \\
\qquad(\boldsymbol{P R I})\end{array}$ & 9 & 37 & Average \\
\hline Block Design & 8 & 25 & Average \\
\hline Matrix Reasoning & 9 & 37 & Average \\
\hline Visual Puzzles & 8 & 25 & Average \\
\hline Figure Weights & 6 & 9 & Low Average \\
\hline $\begin{array}{l}\text { Picture Completion } \\
\text { (Verbal WMI) }\end{array}$ & 11 & 63 & Average \\
\hline Digit Span & 7 & 16 & Low Average \\
\hline Arithmetic & 7 & 16 & Low Average \\
\hline $\begin{array}{l}\text { Letter-Number Seq. } \\
\text { (PSI) }\end{array}$ & 9 & 37 & Average \\
\hline Symbol Search & 7 & 16 & Low Average \\
\hline Coding & 10 & 50 & Average \\
\hline Cancellation & 10 & 50 & Average \\
\hline Mean Subtest Score (15) & 8.47 & 30 & Average \\
\hline
\end{tabular}

Table 3. Wechsler memory scale—fourth edition (WMS-IV) composite scale standard scores.

\begin{tabular}{ccccc}
\hline WMS-IV Index Scores & Index Score & 95\% Confidence Interval & Percentile & Qualitative Description \\
\hline Auditory Memory & 101 & $95-107$ & 53 rd & Average \\
Visual Memory & 117 & $111-122$ & 87 th & High Average \\
Visual Working Memory & 103 & $96-110$ & 58 th & Average \\
Immediate Memory & 113 & $106-119$ & 81 st & High Average \\
Delayed memory & 107 & $100-113$ & 68 th & Average \\
\hline
\end{tabular}


Table 4. Motor functions.

\begin{tabular}{ccccc}
\hline Subtest & Right Hand & Left Hand & Difference & Significance \\
\hline \multicolumn{7}{c}{} & T-Scores & & $\Delta$ T-Score & NS $^{*}$ \\
Finger Tapping & 14 & 25 & -11 & NS \\
Grip Strength & 35 & 37 & -2 & NS \\
Grooved Pegboard & 46 & 48 & -2 & \\
\hline
\end{tabular}

${ }^{*} \mathrm{NS}=$ Nonsignificant; $\mathrm{T}=$ score has a mean of 50 and a standard deviation of 10.

D-KEFS [28] counterpart of the Trail-Making Test: Part B [24] was significantly impaired $(T=30)$. Performance on the Draw-A-Person IQ Test [29] was below expected values for an estimated premorbid FSIQ of 106 and therefore likely impaired (standard score $=76$ ).

The patient scored in the severely depressed range for the Beck Depression Inventory-II [30] (40/63) and in the severe range for the Beck Hopelessness Scale [31] $(15 / 20)$. The patient also exhibited moderate anxiety (23/63) on the Beck Anxiety Inventory [32]. Despite these mood impairments the patient scored in the normal unimpaired range on the Beck Cognitive Insight Scale [33]. Social cognition as measured by the Advanced Clinical Solutions Social Perception battery was normal on Affect Naming, Prosody-Face Matching and ProsodyPair Matching [34]. The MMPI-2-RF demonstrated high emotional and internalizing dysfunction, demoralization, somatic complaints, low positive emotions as well as cognitive and neurological complaints. The patients also demonstrated death ideation, anxiety and social avoidance [35]. The patient's severe depression was treated with a combination of amitriptyline and venlaxafine and she subsequently responded well to pharmacotherapy therapy.

\section{DISCUSSION}

The complete absence of any effects of the pontine lesion on performance of any of the memory measures reinforces the reliance of declarative memory largely on the integrity of medial temporal lobe structures $[24,36]$. See Table 5. Botez-Marquard et al. (2001) examined a single patient with a left cerebellar stroke with comprehensive neuropsychological assessments, single photon emission tomography (SPECT) functional neuroimaging and high resolution structural imaging [37]. Findings included hypoperfusion within the central pons and reduced frontal uptake of radioactive ethylene bis-cysteine mainly within the right frontal lobe. The authors attributed the hypofrontality to diaschisis via fronto-pontine-cerebellar longrange tracts [38]. Botez-Marquard et al. patients' memory was poorer than his intellectual functions unlike our patient where the reverse scenario applied. Daum et al. (1993) postulated that large-scale damage to cholinergic neurons and the locus coeruleus were specifically responsible for memory loss and frontal dysfunction in patients with cerebellar and brainstem lesions [39].

In contrast in our patient the central pons which is close to the serotoninergic median raphe nucleus [3] was the only structure that was damaged. Whereas inspection of MRI images revealed that the noradrenergic anterolateral locus coeruleus nuclei of the upper pons were in all likelihood undamaged [40]. Damage to the raphe nuclei per se, or tracts emanating from it, could explain the myriad of neuropsychiatric features of the patient's illness. Damage to ascending tracts from the raphe nucleus has in particular been implicated in disturbances in sleep-wake cycles [41]. The cholinergic pedunculopontine nucleus of the upper pons and the basal nucleus of Meynert of the basal forebrain were also likely undamaged in our patient [40]. These two cholinergic nuclei are implicated in declarative memory processes associated with the medial temporal lobe [42]. It is possible then that other patients with both cerebellar and pontine lesions after basilar strokes evidence both memory and frontal dysfunction. With intact cholinergic and noradrenergic function due to a highly focalized lesion to the raphe nucleus at the center of the pons memory might be expected to be spared [41]. Indeed several recent studies have shown definitive links between polymorphisms in serotonin transporter genes and serotonin levels and general cognitive ability [43-45].

In summary, the patient's MRI scan close to the neuropsychological evaluation revealed an amorphous central non-enhancing increased T2 signal area in the central pons which was marginally more prominent than when observed with an MRI done 22 months before.

The lesion was adjacent to the fourth ventricle within the pons and revealed the characteristic pontine "batwing" sign [1]. These central pontine myelinolysis lesions can sometimes be accompanied by extrapontine myelotic lesions deep in the cerebral cortical underlying white matter, presumably due to secondary Wallerianlike neurode generation. The spatial configuration and location of the lesion was highly consistent with previously described central pontine myelinolysis - since definitive diagnosis requires MR imaging as opposed to CT imaging. We ruled out a differential diagnosis of extrapontine myelinolysis since a very small centrum semiovale lesion was inconsequential and consistent with normal aging [2].

Ruchinskas (1998) showed that central pontine mye- 
Table 5. Wechsler Memory Scale-Fourth Edition (WMS-IV) subtest age scaled scores. AMI = Auditory Memory Index, VMI = Visual Memory Index, Visual WMI = Visual Working Memory Index.

\begin{tabular}{cccc}
\hline WMS-IV Subtests (AMI) & Age Scaled Score & Percentile & Qualitative Description \\
\hline Logical Memory I & 12 & 75 & High Average \\
Logical Memory II & 10 & 50 & Average \\
Verbal Paired Associates I & 10 & 50 & Average \\
Verbal Paired Associates II & 9 & 37 & Average \\
(VMI) & & & Superior \\
Designs I & 15 & 95 & High Average \\
Designs II & 13 & 84 & Average \\
Visual Reproductions I & 11 & 63 & High Average \\
Visual Reproductions II & 12 & 75 & Average \\
(Visual WMI) & & & High Average \\
Spatial Addition & 8 & 25 & Average \\
Symbol Span & 13 & 64 & 66
\end{tabular}

linolysis (CPM) was associated with a global lowering of many higher cognitive processes on the WAIS in addition to motor deficits presumably as a result of damage to motor tracts within the pons [19]. This researcher hypothesized that CPM also can result in prominent damage to the reticular activating system, disruption of neocortical-ponto-cerebellar tracts and interruption of catecholaminergic pathways. These types of focalized brain stem injuries within the central pons would account for the prominent sleep-wake cycle abnormalities, low levels of general arousal and significant psychiatric morbidity witnessed in the patient's clinical profile. There was a general uniform lowering or flattening of all WAIS-IV scores with the exception of Figure Weights which was disproportionately affected. There was also a notable lowered score on the subtest of verbal abstraction (Similarities: age scaled score $=7$ ). Our patient also did poorly on interpreting proverbs - a good example of a verbal abstraction task on the mental status examination. All three of these tasks are highly correlated with measures of general cognitive ability [24].

The patient's profile included the following: 1) disturbances of consciousness including drowsiness and sleep disorders, 2) dysarthria and difficulties articulating speech especially when fatigued, 3) postural hypotension when standing after sitting or lying down culminating in vertigo, tunnel vision and sensations as if she were going to black out and 4) neuropsychological difficulties including attention, concentration, short-term memory, visual motor and fine motor speed and learning ability. All of these symptoms have been shown to occur in large case series studies of CPM [2]. Severe cases of CPM can result in the locked-in syndrome. Mild cases may demonstrate subtle neuropsychological deficits with more prevalent neuropsychiatric symptoms possibly due to the preponderance of catecholaminergic nuclei and tracts emanating from the pons and involved in emotion, arousal and learning [2].

In this patient there was a discrepancy between the intellectual and memory functions. Memory was unimpaired whereas intellectual functions were generally impaired. Verbal working memory was marginally significantly below nonverbal working memory. Performance was impaired on a measure of constructional praxis (e.g. drawing task), motor dexterity (Finger Tapping test) and motor sequencing (Trails B) consistent with damage to pontine motor tracts. The personality and mood profile was consistent with a diagnosis of severe anxiety and depression probably due to damage to catecholaminergic tracts originating within the pons. Our patient displayed prominent sleep disturbances consistent with reticular activating formation injury.

In summary this patient's central pontine myelinolysis appears to be benign and it is unknown why our patient developed central pontine myelinolysis. It is unclear why some patients can have such abnormalities on structural neuroimaging within the pons and yet continue to function reasonably well. It is possible that studying such focalized lesions within the brainstem with neuropsychological assessments would afford an opportunity to identify cortical-subcortical mutual interactions. In addition to comprehensive neuropsychological evaluations and structural neuroimaging, functional neuroimaging protocols and laboratory computer-based tasks with speed and accuracy measures to study various forms of attention would likely be helpful. That is, if these CPM lesions are transitory there is potentially the basis for understanding before- and after- virtual in vivo lesions in 
otherwise asymptomatic patients with a high degree of resolution with importance for understanding subcortical disorders and dementias. Further studies of such patients with serial structural and functional imaging will be required to determine the hypothesized benign nature of these abnormalities.

\section{ACKNOWLEDGEMENTS}

This research was approved by the Research and Performance Support institutional review committee of the Regina Qu'Appelle Health Region at the Wascana Rehabilitation Centre in Regina, Canada. All research participants provided and their informed written consent in accordance with the Declaration of Helsinki. This study was conducted in accordance with the principles of the Canadian Tri-Council Policy Statement: Ethical Conduct for Research Involving Humans.

We thank the research participant for her involvement in this study. Dr. Gregor Jason provided helpful discussions of the individual participant's neuropsychological evaluations. Joan Harmsworth-Dow of the Wascana Rehabilitation Centre's Health Science Library provided assistance with literature searches. Ms. Marlene Schick and Ms. Mercedes Will kindly provided administrative support during this study. The authors of this article report no conflict of interest.

\section{REFERENCES}

[1] Adams, R.D., Victor, M. and Mancell, E.L. (1959) Central pontine myelinolysis: A hitherto undescribed disease occurring in alcoholic and malnourished patients. Archives of Neurological Psychiatry, 81, 778-780.

[2] Lampl, C. and Yazdi, K. (2002) Central pontine myelinolysis. European Neurology, 47, 3-10. doi:10.1159/000047939

[3] Ropper, A.H. and Samuels, M.A. (2009) Principles of neurology. 9th Edition, McGraw-Hill, New York.

[4] Sterns, R.H., Thomas, D.T. and Herndon, R.M. (1989) Brain dehydration and neurologic deterioration after rapid correction of hyponatremia. Kidney International, 35, 6975. doi:10.1038/ki.1989.9

[5] Kleinschmidt, B.K., Masters,-De, and Norenberg, M.D. (1981) Rapid correction of severe hyponatremia causes demyelinization: Relation to central pontine myelinolysis. Science, 211, 1068-1070. doi:10.1126/science.7466381

[6] Sterns, R.H. (1990) The management of symptomatic hyponatremia. Seminars in Nephrology, 10, 503-504.

[7] Laureno, R. and Illowsky Karp, B. (1997) Myelinolysis after correction of hyponatremia. Annals of Internal Medicine, 126, 57-62.

[8] Vexler, Z.S., Ayus, J.C., Roberts, T.P., Fraser, C.L., Kuchasczyk, J. and Arief, A.I. (1994) Hypoxic and ischemic hypoxia exacerbate brain injury associated with metabolic encephalopathy in laboratory animals. Journal of Clinical Investigation, 93, 256-264. doi:10.1172/JCI116953

[9] Norenberg, M.D. (1983) A hypothesis of osmotic endothelial injury: A pathogenetic mechanisms in central pontine myelinolysis. Archives of Neurology, 40, 66-69. doi:10.1001/archneur.1983.04050020028004

[10] Bonham, C.A., Diminguez, E.A., Fukui, M.B., Paterson, D.L., Pankey, G.A., Wagener, M.M., Fung, J.J. and Singh, N. (1998) Central nervous system lesions in liver transplant recipients: Prospective assessment of indications for biopsy and implications for management. Transplantation, 66, 1596-1604. doi:10.1097/00007890-199812270-00005

[11] Rodriguez, J., Benito-Leon, J., Molina, J.A., Buckels, J. and McMaster, P. (1991) Central pontine myelinolysis associated with cyclosporine in liver transplantation. Neurologia, 13, 437-440.

[12] Bird, G.L., Meadows, J., Goka, J., Polson, R. and Williams, R. (1990) Cyclosporin-associated akinetic mutism and extrapyramidal syndrome after liver transplantation. Journal of Neurology, Neurosurgery, and Psychiatry, 53, 1068-1071. doi:10.1136/jnnp.53.12.1068

[13] Boon, A.P., Carey, M.P., Adams, D.H., Buckels, J. and McMaster, P. (1991) Central pontine myelinolysis in liver transplantation. Journal of Clinical Pathology, 44, 909. 914. doi:10.1136/jep.44.11.909

[14] Odier, C., Nguyen, D.K. and Panisset, M. (2010) Central pontine and extrapontine myelinolysis: From epileptic and other manifestations to cognitive prognosis. Journal of Neurology, 257, 1176-1180. doi:10.1007/s00415-010-5486-7

[15] Mao, S., Liu, Z. and Ding, M. (2011) Central pontine myelinolysis in a patient with epilepsia partialis continua and hyperglycaemic hyperosmolar state. Annals of Clinical Biochemistry, 48, 79-82. doi:10.1258/acb.2010.010152

[16] Vermetten, E., Rutten, S.J., Boon, P.J., Hofman, P.A. and Leentjens, A.F. (1999) Neuropsychiatric and neuropsychological manifestations of central pontine myelinolysis. General Hospital Psychiatry, 21, 296-302. doi:10.1016/S0163-8343(99)00018-3

[17] Joseph, A.P. and Farmer, A. (1995) An unusual case of central pontine myelinolysis. Alcohol, 30, 423-425.

[18] Goebel, H.H. and Herman-Ben Z. P. (1972) Central pontine myelinolysis: A clinical and pathological study of 10 cases. Brain, 14, 495-504. doi:10.1093/brain/95.3.495

[19] Ruchinskas, R. (2008) Cognitive dysfunction after central pontine myelinolysis. Neurocase: The Neural Basis of Cognition, 4, 173-179. doi:10.1212/WNL.43.11.2412-a

[20] Arciero, S., Kempf, C., Bernard, F. and Gosselin, N. (2011) Cognitive and functional performance in daily activities before and after pontine and extrapontine myelinolysis: A case study. Neurocase, 1, 1-7.

[21] Lee, T.M., Cheung, C.C., Lau, E.Y., Mak, A. and Li, L.S. (2003) Cognitive and emotional dysfunction after central pontine myelinolysis. Behavioural Neurology, 14, 103-107.

[22] Price, B.H. and Mesulam, M.M. (1987) Behavioral manifestations of central pontine myelinolysis. Archives of Neurology, 44, 671-673. doi:10.1001/archneur.1987.00520180085025

[23] McCrea, S.M. and Robinson, T.P. (2011) Visual puzzles, figure weights and cancellation: Some preliminary hypotheses on the functional and neural substrates of these three new WAIS-IV subtests. ISRN Neurology, 2011, 19. 
doi: $10.5402 / 2011 / 123173$

[24] Lezak, M.D., Howieson, D.B. and Loring, D.W. (2004) Neuropsychological assessment. 4th Edition, Oxford University Press, New York.

[25] Benson, N., Hulac, D.M. and Kranzler, J.H. (2010) Independent examination of the wechsler adult intelligence scale, 4th edition: What does the WAIS-IV measure. Psychological Assessment, 22, 121-130.

[26] Folstein, M.F., Folstein, S.E. and McHugh, P.R. (1975) Mini-mental state: A practical method for grading the cognitive state if patients for the clinician. Journal of Psychiatric Research, 12, 189-198. doi:10.1016/0022-3956(75)90026-6

[27] Strauss, E., Sherman, E.M. and Spreen, O. (2006) A compendium of neuropsychological tests: Administration, norms and commentary. Oxford University Press, New York.

[28] Delis, D.C., Kaplan, E. and Kramer, J.H. (2001) Deliskaplan executive function system. The Psychological Corporation, San Antonio.

[29] Reynolds, C.R. and Hickman, J.A. (2004) Draw-a-person intellectual ability test for children, adolescents, and adults. Pro-Ed Inc., Austin.

[30] Beck, A.T., Steer, R.A. and Brown, G.K. (1996) Beck depression inventory. 2nd Edition, The Psychological Corporation, San Antonio.

[31] Beck, A.T. and Steer, R.A. (1988) The beck hopelessness scale. The Psychological Corporation, San Antonio.

[32] Beck, A.T. and Steer, R.A. (1993) Beck anxiety inventory. The Psychological Corporation, San Antonio.

[33] Beck, A.T., Baruch, E., Balter, J.M., Steer, R.A. and Warman, D.M. (2004) A new instrument for measuring insight: The Beck cognitive insight scale. Schizophrenia Research, 68, 319-329. doi:10.1016/S0920-9964(03)00189-0

[34] NCS Pearson (2009) Advanced clinical solutions for WAIS-IV and WMS-IV: Administration and scoring manual. The Psychological Corporation, San Antonio.

[35] Ben-Porath, Y.S. and Tellegen, A. (2008) Minnesota Multiphasic personality inventory-2: Restructured form: Manual for administration, scoring and interpretation. Uni- versity of Minnesota Press, Minneapolis.

[36] Heilman, K.M. and Valenstein, E. (2003) Clinical neuropsychology. 4th Edition, Oxford University Press, New York.

[37] Botez-Marquard, T., Bard, C., Leveille, J.and Botez, M.I. (2001) A severe frontal-parietal syndrome following cerebellar damage. European Journal of Neurology, 8, 347353. doi:10.1046/j.1468-1331.2001.00204.x

[38] Rousseaux, M. and Steinling, M. (1992) Crossed hemispheric diaschsis in unilateral cerebellar lesions. Stroke, 23, 511-514. doi:10.1161/01.STR.23.4.511

[39] Daum, I., Ackerman, H., Schugens, M.M., Reimold, C., Dicgans, J. and Birbaumer, N. (1993) The cerebellum and cognitive functions in humans. Behavioral Neuroscience, 107, 411-419. doi:10.1037/0735-7044.107.3.411

[40] Kretschmann, H.J. and Weinrich, W. (2004) Cranial neuroimaging and clinical neuroanatomy: Atlas of MR imaging and computed tomography. 3rd Edition, Thieme, New York.

[41] Kandel, E.R., Schwartz, J.H. and Jessell, T.M. (2000) Principles of neural science. 4th Edition, McGraw-Hill, New York.

[42] Kolb, B. and Whishaw, I.Q. (1996) Fundamentals of human neuropsychology. 4th Edition, W. H. Freeman and Company, New York.

[43] Madsen, K., Erritzoe, D., Mortensen, E.L., Gade, A., Madsen, K., et al. (2011) Cognitive function is related to fronto-striatal serotonin transporter levels-A brain PET study in young healthy subjects. Psychopharmacology (Berlin), 213, 573-581. doi:10.1007/s00213-010-1926-4

[44] Anderson, D.E., Bell, T.A. and Awh, E. (2012) Polymorphisms in the 5-HTTLPR gene mediate storage capacity of visual working memory. Journal of Cognitive Neuroscience, 24, 1069-1076. doi:10.1162/jocn_a_00207

[45] Stollstorff, M., Bean, S.E., Anderson, L.M., Devaney, J.M. and Vaidya, C.J. (2012) Rationality and emotionality: Serotonin transporter genotype influences reasoning bias. Social and Cognitive Affective Neuroscience, in press. 